\title{
Strain-tunable ferroelectricity and its control of Rashba effect in $\mathrm{KTaO}_{3}$
}

\author{
L. L. Tao and J. Wang ${ }^{\text {a) }}$ \\ Department of Physics and The Center of Theoretical and Computational Physics, The University of Hong \\ Kong, Hong Kong, China
}

(Received 28 September 2016; accepted 30 November 2016; published online 16 December 2016)

\begin{abstract}
The effects of epitaxial strain on the ferroelectric, structural properties of $\mathrm{KTaO}_{3}$ are studied by means of first-principles calculations. We show that the ferroelectric polarization magnitude as well as the orientation can be tuned by an in-plane strain: the $c$-phase is energetically more stable than the $a a$-phase at a large compressive strain while a phase transition from $c$ - to $a a$-phase is observed at a large tensile strain, owing to the significant polarization-strain coupling. More importantly, based on relativistic first-principles calculations, we demonstrate a large Rashba spin splitting in the strained $\mathrm{KTaO}_{3}$. Interestingly, the spin textures in momentum space can be controlled and switched via polarization switching. Our tight-binding analysis indicates that the combination of spin-orbit coupling and ferroelectric distortion plays a key role for the observed Rashba spin splitting. Our results present some fundamental understanding of the interplay between Rashba effect and ferroelectricity in oxides and open avenues for nonvolatile spintronic device applications. Published by AIP Publishing. [http://dx.doi.org/10.1063/1.4972198]
\end{abstract}

\section{INTRODUCTION}

The Rashba effect, characterized by the momentumdependent spin splitting of energy levels, has recently aroused a great interest due to the fascinating physics it displayed as well as the potential applications in spintronic devices. ${ }^{1}$ Generally, the Rashba effect is found on the surface of heavy metals, ${ }^{2}$ e.g., $\mathrm{Au}(111)^{3,4}$ and $\operatorname{Ir}(111),{ }^{5}$ the surface of oxide, e.g., $\operatorname{SrTiO}_{3}(001),{ }^{6}$ and heterojunction interfaces, e.g., InGaAs/InAlAs. ${ }^{7}$ Recently, a sizable Rashba effect was also observed in bulk materials with inversion asymmetry, such as BiTeI, ${ }^{8}$ ferroelectric (FE) $\mathrm{GeTe}^{9}$ and ferroelectric organic perovskites. ${ }^{10-12}$ It is known that the presence of both time-reversal symmetry $\epsilon_{k \uparrow}=\epsilon_{-k \downarrow}\left(\epsilon_{k \uparrow, \downarrow}\right.$ eigenvalues) and spatial inversion symmetry $\epsilon_{k \uparrow}=\epsilon_{-k \uparrow}$ leads to the spin degeneracy $\epsilon_{k \uparrow}=\epsilon_{k \downarrow}$. The Rashba-like spin splitting can thus be expected in those materials with spatial inversion symmetry broken, namely, the potential $V(\vec{r}) \neq V(-\vec{r})$. Among those materials, FE materials are promising candidates to be exploited as novel spintronic devices due to the nonvolatile property of FE polarization as well as potential all-electric control of spin. Recently, giant Rashba effect was theoretically predicted in the FE semiconductor GeTe, and the spin textures can be fully controllable and switchable via the polarization switching. ${ }^{9}$ The similar phenomenon was also reported in other FE materials. ${ }^{10-14}$ In this regard, the FE Rashba semiconductors or insulators as a kind of novel multifunctional materials ${ }^{15}$ can be used to design new types of devices, such as nonvolatile spin-fieldeffect transistor ${ }^{9,16}$ and ferroelectric tunnel junctions. ${ }^{17-19}$

It would be beneficial to find FE Rashba materials with both large polarization and strong spin-orbit coupling (SOC) for realization of controlling the spin via an electric field. Here we consider an appealing perovskite oxide- $\mathrm{KTaO}_{3}$ (KTO). First, it was shown in the experiment that the

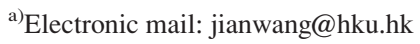

strained KTO films exhibit significant $\mathrm{FE}$ effects. ${ }^{20}$ Secondly, unlike the widely used lead-related $\mathrm{PbTiO}_{3}$, the lead-free KTO is one of the environment-friendly FE materials. Thirdly, KTO is a polar oxide that the two-dimensional electron (hole) gas can be naturally formed in the $\mathrm{TaO}-$ terminated (KO-terminated) surface, which makes it a promising candidate for the field-effect transistor. ${ }^{21}$ The surface Rashba effect due to the structure inversion symmetry broken has been experimentally observed ${ }^{22}$ and theoretically investigated. ${ }^{23}$ More interestingly, it was shown that the surface Rashba effect of KTO can be tuned by an electric field. ${ }^{23}$ Finally, the atomic SOC is expected to be large in KTO due to the presence of heavy $5 d$ element Ta. To our knowledge, the Rashba effect in bulk KTO due to the FE distortion which naturally breaks the inversion symmetry has not been explored. It is the purpose of this paper to investigate the strain-tunable ferroelectricity and especially the interplay between Rashba effect and ferroelectricity in KTO. Compared with the electric field tunable Rashba effect, the FE control of Rashba effect is nonvolatile and more easily amenable.

Here, based on first-principles calculations, we have investigated the strain-tunable ferroelectric polarization: the most energetically stable phase of KTO under different in-plane strains has been identified and the corresponding polarization was calculated. A large Rashba-like spin splitting in the strained KTO was observed. We also show that spin textures can be fully controllable by the FE polarization. The microscopic physics for FE-driven Rashba effect has been analyzed from a tight-binding (TB) model calculation.

\section{COMPUTATIONAL METHOD AND DETAILS}

Our atomic and electronic structure calculations were performed using the plane-wave ultrasoft pseudopotential method $^{24}$ implemented in the Quantum Espresso package. ${ }^{25}$ 
An energy cutoff of $680 \mathrm{eV}$ and local density approximation $(\mathrm{LDA})^{26}$ for the exchange and correlation functional were used throughout. Atomic relaxation was performed using a $10 \times 10 \times 10 k$-point mesh until the forces on each atom were smaller than $2.6 \mathrm{meV} / \AA$. A dense, $20 \times 20 \times 20, k$ point mesh was used for the self-consistent calculations to obtain the total energies and electronic structures. We evaluated the FE polarization $P$ by using ${ }^{27,28}$

$$
P=\frac{1}{\Omega} \sum_{m} Z_{m}^{*} \delta z_{m},
$$

where $\Omega$ is the unit-cell volume, $Z_{m}^{*}$ the Born effective charge of the $m$ th atom, and $\delta z_{m}$ the displacement of the $m$ th atom. The Born effective charges $Z_{m}^{*}$ were calculated using density functional perturbation theory. ${ }^{29} Z_{m}^{*}$ for $\mathrm{K}, \mathrm{Ta}, \mathrm{O}_{\perp}$ ( $\mathrm{TaO}$ plane) and $\mathrm{O}_{\|}$(KO plane) ions in cubic KTO phase $\left(a_{0}=3.94 \AA\right)$ are, respectively, $1.15,8.62,-1.69$, and -6.41 electrons. Note that the calculated $a_{0}=3.94 \AA$ agrees well with the previous results using LDA function. ${ }^{20,30}$

\section{RESULTS AND DISCUSSION}

\section{A. Strain-tunable ferroelectricity}

We first present the strain tunable structural and FE properties. The epitaxial strain provides an efficient way to design artificial structures, which reveal rich physical properties. For example, paraelectric (PE)-to-ferroelectric transition in $\mathrm{SrTiO}_{3}$ films can be triggered by an epitaxial strain. ${ }^{31,32}$ Fig. 1(a) shows the total energies and $c / a$ ratios of bulk KTO under different strains. The in-plane strain is defined as $\left(a-a_{0}\right) / a_{0}$, where $a\left(a_{0}\right)$ is the in-plane lattice constant of tetragonal (cubic) KTO. Fig. 1(b) shows the values of polarization for different phases as a function of strain. To find the most stable phase at each strain, we fully relaxed the atomic positions as well as the out-of-plane lattice constants starting from three possible phases: ${ }^{33}$ (i) the paraelectric $p$ phase $\left(P_{x}=P_{y}=P_{z}=0\right)$, (ii) the $c$ phase $\left(P_{x}=P_{y}=0, P_{z} \neq 0\right)$, and (iii) the $a a$ phase $\left(P_{x}=P_{y} \neq 0, P_{z}=0\right)$ and identified the minimum energy phase. First, the energy differences between different phases are negligible at small strains, indicating that no phase transition occurs, and KTO is in the stable $p$ phase. The strain dependency of $c / a$ is almost linear. As the tensile strain increases (exceeds $0.5 \%$ ), a phase transition from $p$ to $a a$ phase occurs, characterized by the lower total energy and sizable polarization value $P_{110}$. On the other hand, with increase of the compressive strain (exceeds 1\%), the stable $c$ phase appears, and a large polarization value $P_{001}$ is observed. Note that the similar strain-tunable phase transition was also predicted in strained $\mathrm{BaZrO}_{3}$ by firstprinciples calculations. ${ }^{34}$ At large compressive (tensile) strain, the increase (decrease) of $c / a$ is significant. The polarization becomes larger than $40 \mu \mathrm{C} / \mathrm{cm}^{2}$ at the compressive (tensile) strain of $-3.5 \%$ (3\%), which is comparable to that of the strained $\mathrm{BaTiO}_{3}{ }^{19}$ It can be seen that an epitaxial strain can induce rich phase transitions and provide an efficient way to tune the polarization of KTO.
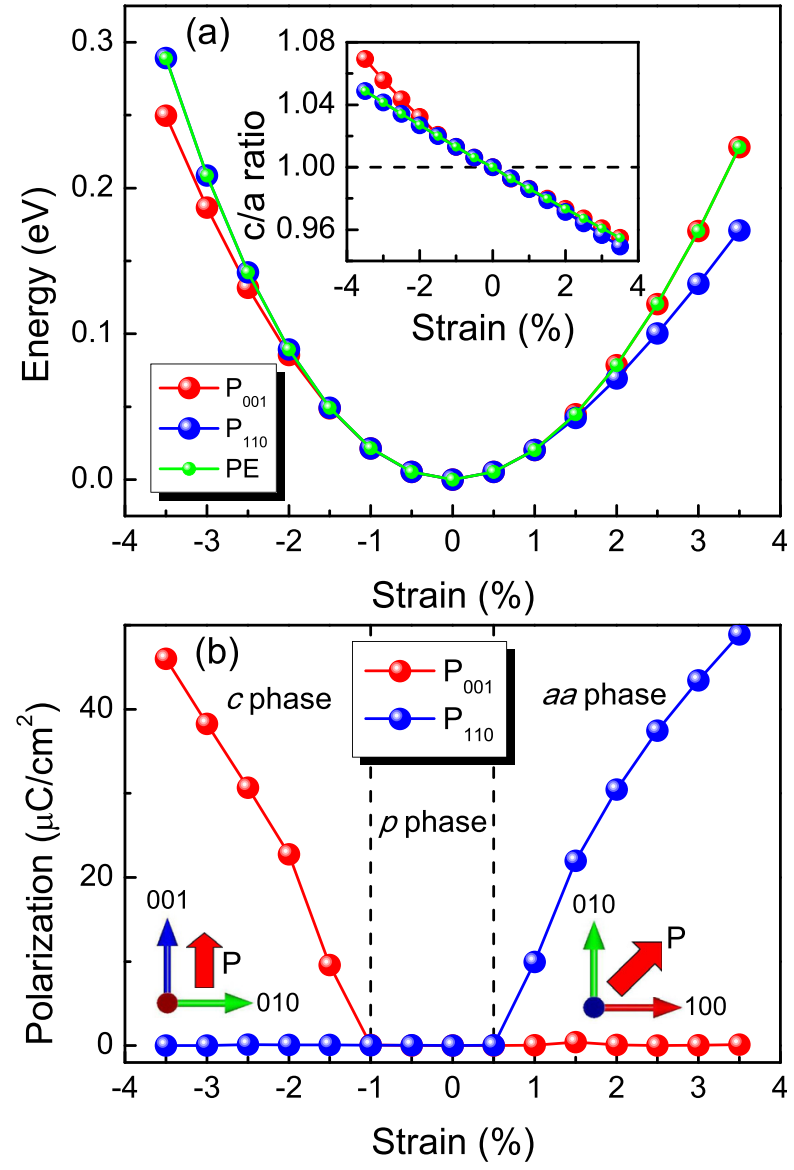

FIG. 1. (a) Total energy versus the strain for KTO with different initial polarization states. Inset: strain dependence of relaxed $c / a$ ratios. (b) Strain dependence of FE polarization.

\section{B. Rashba effect}

We now investigate the Rashba effect in FE KTO. Fig. 2(a) shows the crystal structure and first Brillouin zone of $c$ phase KTO induced by the compressive strain of $-3.0 \%$. Fig. 2(b) shows the fully relativistic band structure. We see that KTO is an indirect-band-gap insulator that the valenceband maximum (VBM) and conduction-band minimum (CBM) are located at the $M$ and $\Gamma$ point, respectively. Clearly, the spin splittings of bands along $\Gamma-X$ and $\Gamma-M$ directions are pronounced and are suppressed along the $\Gamma-Z$ direction. As highlighted by the red box, a zoom of conduction bands around the $\Gamma$ point reveals a Rashba-like band splitting, characterized by the distinct momentum offset. For those bands with a larger spin splitting effect, we calculate the expectation values of the spin operators $s_{\alpha}$ $(\alpha=x, y, z)$ on the spinor wave-functions $\psi_{k}$, namely, $s_{\alpha}=\frac{\hbar}{2}$ $\left\langle\psi_{k}\left|\sigma_{\alpha}\right| \psi_{k}\right\rangle$. The in-plane spin textures $\left(s_{x}\right.$ and $s_{y}$ components) of CBM bands near the $\Gamma$ point are shown in Figs. 2(c) and 2(d), while the $s_{z}$ component is negligible. The two branch bands show clearly the Rashba-like features with the spin orientation perpendicular to the momentum direction and the outer and inner branch bands having opposite spin textures. Intriguingly, the spin textures are fully reversed upon switching the polarization as evident by comparing Figs. 2(c) and 2(d). As for the $a a$-phase, KTO induced by a tensile strain, the polarization axis changes from [001] to 

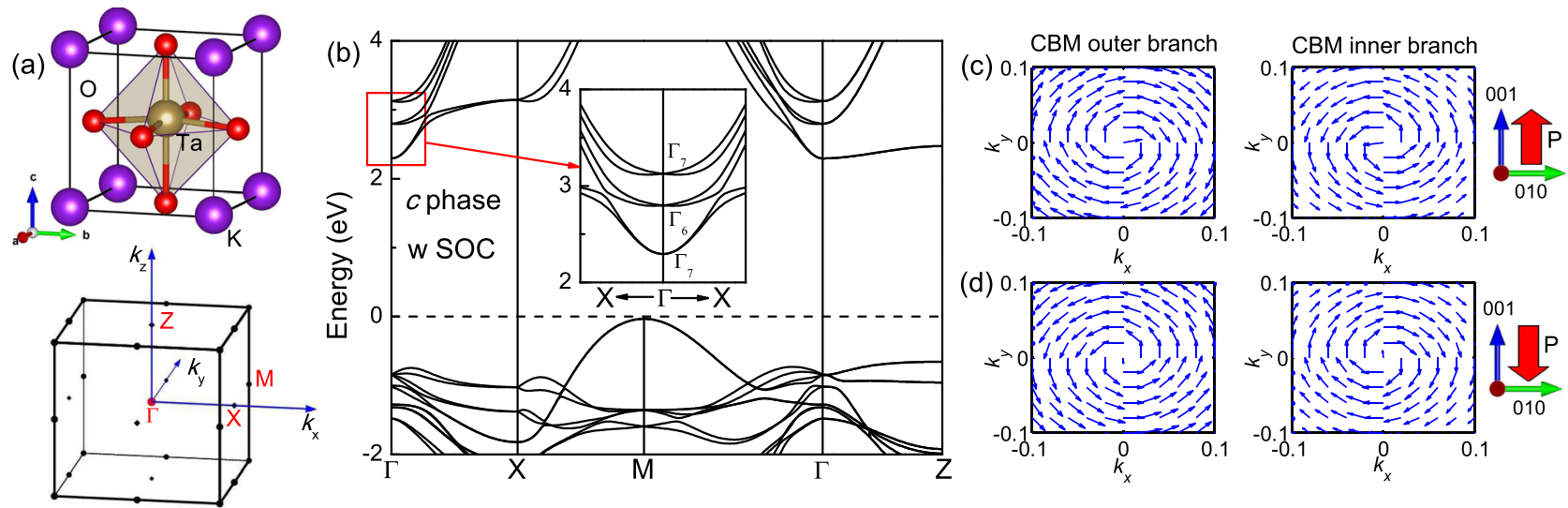

FIG. 2. (a) Crystal structure and first Brillouin zone of bulk KTO. (b) Band structure with SOC for $c$-phase KTO ( $-3.0 \%$ compressive strain) along the high symmetry lines $\Gamma\left(\begin{array}{lll}0 & 0 & 0\end{array}\right)-\mathrm{X}\left(\begin{array}{lll}0.5 & 0 & 0\end{array}\right)-\mathrm{M}\left(\begin{array}{lll}0.5 & 0.5 & 0\end{array}\right)-\Gamma\left(\begin{array}{lll}0 & 0 & 0\end{array}\right)-\mathrm{Z}\left(\begin{array}{lll}0 & 0 & 0.5\end{array}\right)$. Inset: zoom of the band structure in the red box. ((c) and (d)) In-plane spin textures $\left(s_{x}\right.$ and $s_{y}$ components) near the $\Gamma$ point for the CBM outer (left) and inner (right) branches. (c) for up polarization case and (d) for down polarization case, as denoted by the red arrows.

[110] and the similar polarization control of spin textures is expected. Indeed, as shown in Fig. 3 for the KTO with a compressive strain of $-3.0 \%, s_{z}$ components are opposite for the inner and outer branch band and can be fully reversed upon polarization reversal. Note that the spin textures are in the plane perpendicular to polarization axis and the appearance of $s_{z}$ components is a natural consequence for the $a a$-phase KTO. Therefore, it is well established that the polarization control of spin textures can be achieved in the strained KTO.

The above spin textures reversal due to the polarization switching can be explained from a model Hamiltonian as well as a symmetry argument. Taking the $c$-phase KTO as an example, by including the Rashba SOC, we consider the Hamiltonian as follows:

$$
\mathcal{H}=\frac{p_{x}^{2}+p_{y}^{2}}{2 m_{x y}^{*}}+\frac{p_{z}^{2}}{2 m_{z}^{*}}+\frac{\alpha_{R}}{\hbar}(\vec{p} \times \hat{z}) \cdot \vec{\sigma},
$$

where $\alpha_{R}$ is the well-known Rashba coefficient, $\hbar$ the reduced Planck's constant, $\vec{p}=\left(p_{x}, p_{y}, p_{z}\right)=\hbar \vec{k}$ the electron
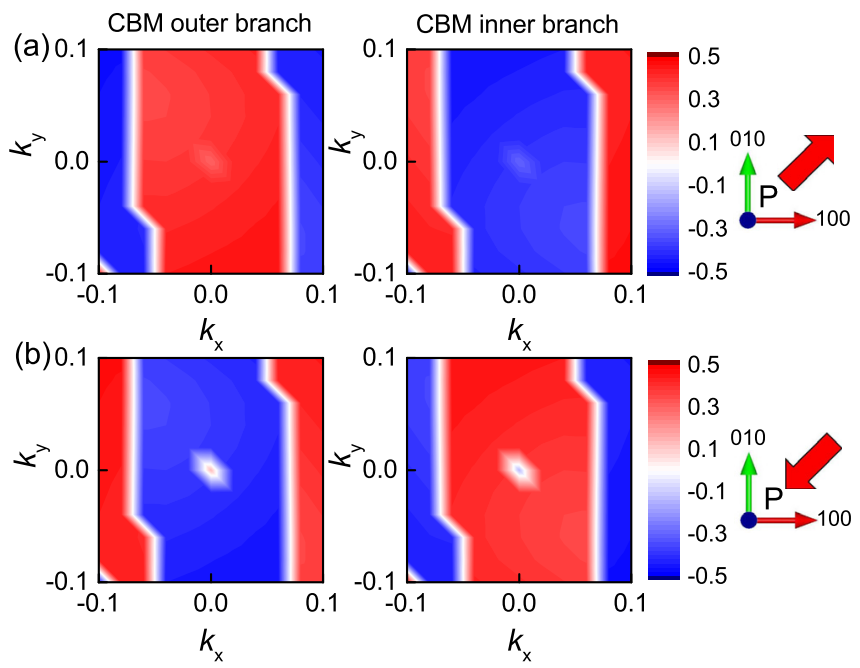

FIG. 3. Spin distribution ( $s_{z}$ component) near the $\Gamma$ point for the CBM outer (left) and inner (right) branches. (a) for [110] polarization case and (b) for [11 10$]$ polarization case, as denoted by the red arrows. The bulk KTO is at the $a$ a phase induced by a tensile strain of $3.0 \%$. momentum, $m_{x y}^{*}\left(m_{z}^{*}\right)$ the electron mass in the $x y$ plane (along the $z$ axis), and $\vec{\sigma}=\left(\sigma_{x}, \sigma_{y}, \sigma_{z}\right)$ the Pauli spin matrices. For $c$-phase KTO, the polarization is along the $z$ axis along which the inversion symmetry is broken. We find that the eigenenergies $\epsilon_{k}$ and eigenstates $\psi_{k}$ are, respectively, given by

$$
\begin{aligned}
& \epsilon_{k}^{ \pm}=\frac{\hbar^{2}}{2 m_{x y}^{*}}\left(k_{x}^{2}+k_{y}^{2}\right)+\frac{\hbar^{2}}{2 m_{z}^{*}} k_{z}^{2} \pm\left|\alpha_{R}\right| \sqrt{k_{x}^{2}+k_{y}^{2}}, \\
& \psi_{k}^{ \pm}=\frac{1}{\sqrt{2}}\left(\begin{array}{c} 
\pm \frac{\alpha_{R} \mid}{\alpha_{R}} e^{i \gamma_{\mathbf{k}}} \\
1
\end{array}\right) e^{\vec{k} \cdot \vec{r}},
\end{aligned}
$$

where $\tan \left(\gamma_{k}\right)=\frac{k_{x}}{k_{y}}$. Taking the upper branch $\left(\epsilon_{k}^{+}, \psi_{k}^{+}\right)$as an example, it is straightforward to obtain the expectation value of the spin operators $\sigma_{\alpha}$

$$
\bar{\sigma}_{x}=\frac{\left|\alpha_{R}\right|}{\alpha_{R}} \cos \gamma_{k}, \quad \bar{\sigma}_{y}=-\frac{\left|\alpha_{R}\right|}{\alpha_{R}} \sin \gamma_{k}, \quad \bar{\sigma}_{z}=0 .
$$

We see that the spin orientations are determined by the Rashba coefficient $\alpha_{R}$, which is directly related to the potential gradient resulting from FE distortion. One can thus anticipate that the change of the sign of $\alpha_{R}$ induced by switching polarization will result in the reversal of spin textures in the plane perpendicular to the polarization axis.

From the symmetry point of view, the reversal of spin textures induced by the polarization switching does not depend on the specific form of Hamiltonian. ${ }^{11}$ This is due to the fact that $\left\langle\psi_{\vec{k}}(-\vec{P})|\vec{\sigma}| \psi_{\vec{k}}(-\vec{P})\right\rangle=-\left\langle\psi_{\vec{k}}(+\vec{P})|\vec{\sigma}| \psi_{\vec{k}}(+\vec{P})\right\rangle .^{11}$

We now analyze the physical origin of Rashba spin splittings observed in $c$-phase KTO from a tight-binding Hamiltonian. Take the case of $-3.0 \%$ strain, for example, we consider the following Hamiltonian: ${ }^{35-38}$

$$
\mathcal{H}=\mathcal{H}_{0}+\mathcal{H}_{S O}+\mathcal{H}_{\gamma}
$$

where the first and second terms are hopping and atomic SOC Hamiltonian, respectively. The third term arises due to the inversion symmetry broken. We consider the band dispersion along $\Gamma-X$ direction, which is in the plane 

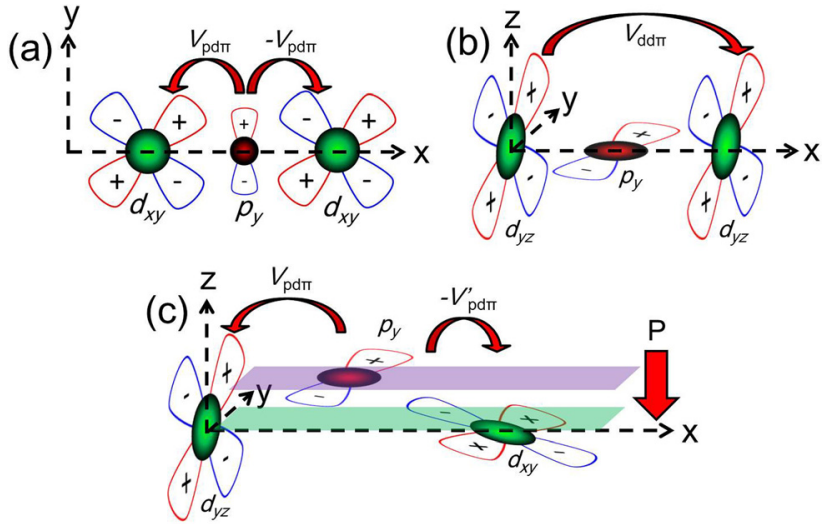

FIG. 4. A sketch of hopping process. (a) $\pi_{p d}$ hopping process between Ta- $d$ and O- $p$ orbitals, (b) $\pi_{d d}$ hopping process between Ta- $d$ orbitals, and (c) $\pi_{p d}$ hopping process induced by FE distortion as denoted by the red arrow. The dark green and red circles denote the Ta and $\mathrm{O}$ atoms, respectively.

perpendicular to the polarization axis. The hopping Hamiltonian $\mathcal{H}_{0}$ in the Ta- $t_{2 g}\{|y z\rangle,|z x\rangle,|x y\rangle\}$ basis set reads

$$
\begin{aligned}
\mathcal{H}_{0} & =\left(\begin{array}{ccc}
\epsilon_{y z} & 0 & 0 \\
0 & \epsilon_{z x} & 0 \\
0 & 0 & \epsilon_{x y}
\end{array}\right) \otimes \sigma_{0}, \\
\epsilon_{y z} & =-2 t^{\prime} \cos k_{x}-2 t \cos k_{y}, \\
\epsilon_{z x} & =-2 t \cos k_{x}-2 t^{\prime} \cos k_{y}, \\
\epsilon_{x y} & =\Delta-2 t \cos k_{x}-2 t \cos k_{y}
\end{aligned}
$$

where $t$ and $t^{\prime}$ are the hopping amplitudes between Ta- $t_{2 g}$ orbitals and $\sigma_{0}$ is the $2 \times 2$ unit matrix. $t$ is the dominant $\pi_{p d}$ hopping, namely, $t=\frac{V_{p d \pi}^{2}}{\Delta_{p d}}$, where $V_{p d \pi}$ denotes the $\pi$-bond hopping between Ta- $d$ and O-p orbitals, and $\Delta_{p d}$ is the corresponding energy difference. $t^{\prime}$ describes the weaker $\pi_{d d}$ hopping. The hopping processes are schematically illustrated in Figs. 4(a) and 4(b). $\Delta$ denotes the energy difference between the $d_{x y}$ orbital and the degenerate $d_{y z / z x}$ orbital. The atomic SOC Hamiltonian $\mathcal{H}_{S O}$ is given by ${ }^{35}$

$$
\mathcal{H}_{S O}=\lambda_{S O}\left(\begin{array}{cccccc}
0 & 0 & i & 0 & 0 & -1 \\
0 & 0 & 0 & -i & 1 & 0 \\
-i & 0 & 0 & 0 & 0 & i \\
0 & i & 0 & 0 & i & 0 \\
0 & 1 & 0 & -i & 0 & 0 \\
-1 & 0 & -i & 0 & 0 & 0
\end{array}\right)
$$

in the $\{|y z\rangle,|z x\rangle,|x y\rangle\} \otimes\{\uparrow, \downarrow\}$ basis set, where $\lambda_{S O}$ is the atomic SOC strength. $\mathcal{H}_{\gamma}$ comes from the FE distortion and can be expressed as ${ }^{36}$

$$
\mathcal{H}_{\gamma}=2 \gamma\left(\begin{array}{ccc}
0 & 0 & -i \sin k_{x} \\
0 & 0 & -i \sin k_{y} \\
i \sin k_{x} & i \sin k_{y} & 0
\end{array}\right) \otimes \sigma_{0}
$$

in the $\{|y z\rangle,|z x\rangle,|x y\rangle\}$ basis set. where $\gamma$ is the hopping integral induced by FE polarization, $\gamma=\frac{V_{p d \pi} V_{p d \pi}^{\prime}}{\Delta_{p d}}$ and the hopping process is schematically illustrated in Fig. 4(c).

Fig. 5 shows the comparison between the DFT and tight-binding band structures for $c$-phase KTO $(-3.0 \%$ strain). All tight-binding parameters obtained from the best fit to the DFT results are summarized in Table I. We see that $t\left(t^{\prime}\right)$ is relatively reduced (increased) in $c$-FE structure
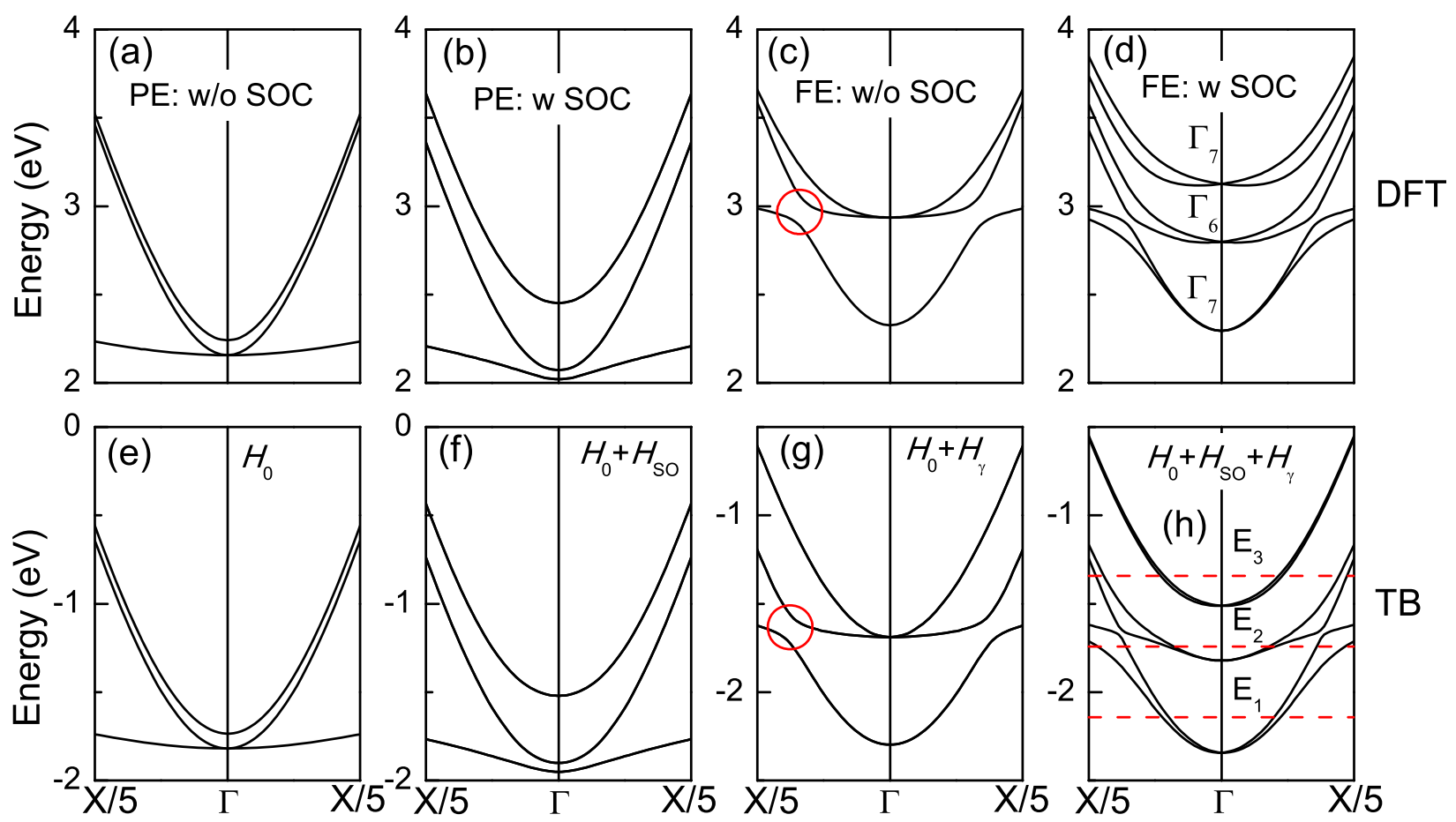

FIG. 5. ((a)-(d)) DFT band structures for $c$-phase KTO (-3.0\% strain) along the $\Gamma-X$ direction of Ta- $t_{2 g}\left(d_{y z}, d_{z x}, d_{x y}\right)$ orbitals for $($ a) paraelectric $(\mathrm{PE})$ case without (w/o) SOC, (b) PE case with (w) SOC, (c) ferroelectric (FE) case without (w/o) SOC. (d) FE case with (w) SOC. The symmetries at the $\Gamma$ point for different bands are denoted. ((e)-(h)) Tight-binding (TB) band structures of Ta- $t_{2 g}$ orbitals obtained with different Hamiltonian forms. The red circles in (c) and (g) highlight the band gap arising from $d_{y z}-d_{x y}$ mixing induced by FE distortion. 
TABLE I. Tight-binding parameters (in units of eV) for bulk KTO in PE ( $c$ and $a a$ phase) and FE ( $c$ phase) structures. Those parameters are obtained from best fits to the DFT results.

\begin{tabular}{lccccc}
\hline \hline Structure & $\boldsymbol{t}$ & $\boldsymbol{t}^{\prime}$ & $\boldsymbol{\Delta}$ & $\boldsymbol{\lambda}_{\text {so }}$ & $\boldsymbol{\gamma}$ \\
\hline$c$-PE & 0.852 & 0.058 & 1.671 & 0.133 & 0 \\
$c$ - $\mathrm{FE}$ & 0.781 & 0.063 & 0.827 & 0.133 & 0.05 \\
$a a-\mathrm{PE}$ & 0.671 & 0.035 & 1.206 & 0.133 & 0 \\
\hline \hline
\end{tabular}

compared to those in the $c$-PE structure. In addition, $t, t^{\prime}$ and $\Delta$ are slightly reduced in $a a-\mathrm{PE}$ structure compared to those in the $c$-PE one. Note that $\gamma$ is proportional to the magnitude of FE polarization as expected from the increase of hopping integral between the Ta- $d$ and O-p orbitals. Fig. 5(a) shows the band structure for paraelectric (PE) case without SOC, we see that, at the $\Gamma$ point, the three-fold degenerate $t_{2 g}$ $\left(d_{y z}, d_{z x}, d_{x y}\right)$ orbitals are split into two-fold degenerate $\Gamma_{6}$ $\left(d_{y z}, d_{z x}\right)$ state and a $\Gamma_{7}\left(d_{x y}\right)$ state under the $C_{4 v}$ crystal field. The two-fold degenerate $\Gamma_{6}$ state is further split into a $\Gamma_{7}$ $\left(d_{x z}\right)$ state and a $\Gamma_{6}\left(d_{y z}\right)$ state in the presence of SOC; even so, the band is however still not spin splitting, as shown in Fig. 5(b). In the case of FE without SOC shown in Fig. 5(c), the band exhibits a state-mixing (between $d_{y z}$ and $d_{x y}$ ) induced band gap as highlighted by the red circles and the parameter $\gamma$ in Eq. (8) is determined from the best fit to this band gap. This is expected due to the hopping between $d_{y z}$ and $d_{x y}$ (see Eq. (8)) orbitals induced by FE distortion, as schematically shown in Fig. 4(c). Fig. 5(d) shows the band structure for FE case with SOC, the most striking feature is the distinct band spin splittings. We conclude that the combination of FE distortion and SOC gives rise to the observed band spin splittings. Figs. 5(e)-5(h) shows the Ta- $t_{2 g}\left(d_{y z}\right.$, $d_{z x}, d_{x y}$ ) tight-binding (TB) band structures. Overall, the TB model reproduces those DFT results very well. Again, we see that the band spin splittings only occur due to the coexistence of FE distortion and SOC, which agrees well with the DFT results. The combined effect of FE distortion and atomic SOC yielding the Rashba spin splittings was also reported in the $\mathrm{BaTiO}_{3} / \mathrm{BaOsO}_{3}$ heterostructure. ${ }^{38}$

Now we investigate the Rashba spin splitting in the momentum space. From the tight-binding Hamiltonian of Eq. (5), the spin-resolved density of states (DOS) $s_{\alpha}\left(E, k_{x}, k_{y}\right)(\alpha=x, y, z)$ can be calculated as

$$
s_{\alpha}\left(E, k_{x}, k_{y}\right)=-\frac{\hbar}{2 \pi} \operatorname{Tr}\left\{\operatorname{Im}\left[\sigma_{\alpha} G^{r}\left(E, k_{x}, k_{y}\right)\right]\right\},
$$

where $\sigma_{\alpha}$ is the Pauli spin matrices, and the $\operatorname{Tr}$ denotes the trace over the $t_{2 g}$ orbitals. The retarded Green's function $G^{r}\left(E, k_{x}, k_{y}\right)$ is given by

$$
G^{r}\left(E, k_{x}, k_{y}\right)=\left[E+i \eta-H\left(k_{x}, k_{y}\right)\right]^{-1},
$$

where $\eta$ is an infinitesimal energy. Fig. 6 shows the in-plane spin DOS $s_{\|}=\left(s_{x}, s_{y}\right)$ distributions corresponding to different energy cuts. For an energy cut $\left(E_{1}\right)$ near the CBM as shown in Fig. 6(a), the spin splitting is nearly isotropic in momentum space, and the spin is locked to the momentum revealing the nature of Rashba spin splitting, as evident from the circular shapes and green arrows. As we shall see later, the analytic effective Hamiltonian near the CBM is a Rashba form and the Rashba parameter is isotropic. For a higher energy cut $\left(E_{2}\right)$ in Fig. $6(\mathrm{~b})$, the spin splitting is anisotropic with a large splitting along the $\Gamma-X$ direction while the splitting is reduced along the $\Gamma-M$ direction. As expected, the anisotropic splitting reflects the four-fold rotational symmetry of tetragonal KTO whose point group is $C_{4 v}\left(C_{4}\right.$ axis is along the [001] direction). For an energy cut $E_{3}$ in Fig. $6(\mathrm{c})$, the anisotropic splitting is also observed, and the splitting is enhanced along the $\Gamma-M$ direction compared with that along the $\Gamma-X$ direction.

As stated above, the Rashba spin splitting is nearly isotropic near the CBM. In the following, we derive an effective Hamiltonian for the energy around the CBM. The effective Hamiltonian can be obtained by using L $\ddot{o}$ wdin downfolding technique. ${ }^{23,39}$ Namely we can rewrite the Hamiltonian of Eq. (5) in the form

$$
\mathcal{H}=\mathcal{H}_{0}+\mathcal{H}_{S O}+\mathcal{H}_{\gamma}=\left(\begin{array}{cc}
h_{1} & \beta \\
\beta^{\dagger} & h_{0}
\end{array}\right),
$$

where

$$
\begin{aligned}
& h_{0}=\left(\begin{array}{cc}
\epsilon_{x y} & 0 \\
0 & \epsilon_{x y}
\end{array}\right) \text {, } \\
& h_{1}=\left(\begin{array}{cccc}
\epsilon_{y z} & 0 & i \lambda_{S O} & 0 \\
0 & \epsilon_{y z} & 0 & -i \lambda_{S O} \\
-i \lambda_{S O} & 0 & \epsilon_{z x} & 0 \\
0 & i \lambda_{S O} & 0 & \epsilon_{z x}
\end{array}\right) \text {, } \\
& \beta=\left(\begin{array}{cc}
-i 2 \gamma \sin k_{x} & -\lambda_{S O} \\
\lambda_{S O} & -i 2 \gamma \sin k_{x} \\
-i 2 \gamma \sin k_{y} & i \lambda_{S O} \\
i \lambda_{S O} & -i 2 \gamma \sin k_{y}
\end{array}\right) .
\end{aligned}
$$
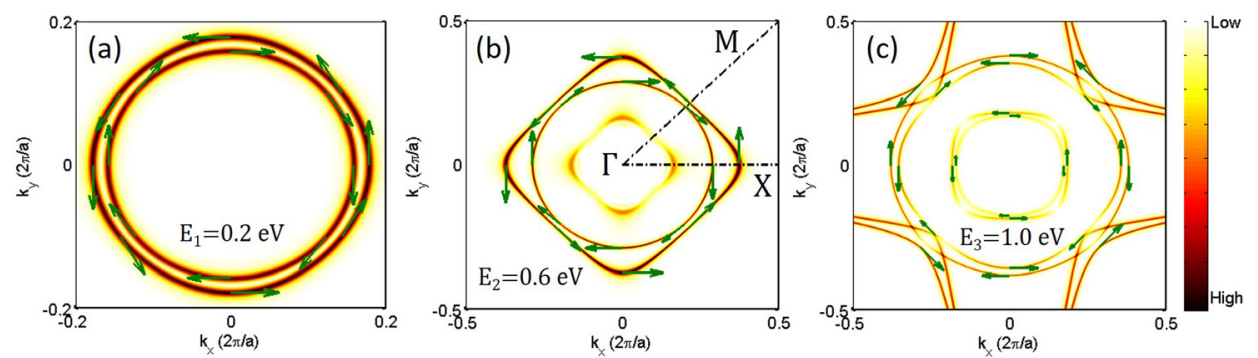

FIG. 6. In-plane spin $s_{\|}=\left(s_{x}, s_{y}\right)$ distributions corresponding to an energy cut at (a) $E_{1}=0.2 \mathrm{eV}$, (b) $E_{2}=0.6 \mathrm{eV}$, and (c) $E_{3}=1.0 \mathrm{eV}$ above the CBM as indicated by the red dashed lines in Fig. $5(\mathrm{~g})$. The directions are indicated by the green arrows for representative points while the magnitude $\left|s_{\|}\right|$is presented by the color coding. The energy broadening $\eta=0.01$ in our numerical calculations. 
The effective Hamiltonian $H_{\text {eff }}$ in the $h_{0}$ subspace can be expressed as ${ }^{39}$

$$
H_{e f f}=h_{0}+\beta^{\dagger}\left(E-h_{1}\right)^{-1} \beta .
$$

For small $k$, retaining the terms up to linear $k$, we get

$$
H_{e f f}=\Delta-4 t+\frac{2 \lambda_{S O}^{2}}{\Delta-2\left(t-t^{\prime}\right)}+\frac{4 \gamma \lambda_{S O}}{\Delta-2\left(t-t^{\prime}\right)}\left(k_{x} \sigma_{y}-k_{y} \sigma_{x}\right) .
$$

The third term in $H_{\text {eff }}$ can be rewritten as the familiar Rashba form, namely, $\alpha_{R}(\vec{k} \times \hat{z}) \cdot \vec{\sigma}$, with the Rashba coefficient $\alpha_{R}=\frac{4 \gamma \lambda_{S O}}{\Delta-2\left(t-t^{\prime}\right)}$. We see that the Rashba coefficient is $k$ independent, that is, being isotropic in momentum space. This is in line with the numerical result in Fig. 5(a). Moreover, $\alpha_{R}$ is in proportion to $\gamma$ which is directly related to the FE polarization, indicating the feasibility of FE controlling Rashba effect. The fact that $\alpha_{R}$ in proportion to $\gamma \lambda_{S O}$ is in line with that the Rashba effect is due to the combined effect of SOC and FE distortion.

\section{CONCLUSIONS}

In summary, we have demonstrated the coexistence of large FE polarization and Rashba effect in the strained KTO through the first-principles calculations. More intriguingly, the spin textures can be switchable via polarization switching. This enables the control of spin via an electric field, which can be used to design the nonvolatile spin-field-effect transistor. ${ }^{9}$ The analysis from a tight-binding model indicates that both the FE polarization and SOC play crucial roles for the observed large Rashba spin spitting. Both the isotropic and anisotropic Rashba spin splittings in momentum space were observed from a tight-binding calculation. Our results provide an insight into the nature of FE-Rashba coupling in oxides and suggest an appealing FE Rashba material that can be exploited as novel all-electric spintronic devices.

\section{ACKNOWLEDGMENTS}

This work is supported by the University Grant Council of Hong Kong (Contract No. AoE/P-04/08) and the National Natural Science Foundation of China (Grant No. 11374246). We are grateful to the National Supercomputer Center in Tianjin for providing the computational facility.

\footnotetext{
${ }^{1}$ A. Manchon, H. C. Koo, J. Nitta, S. M. Frolov, and R. A. Duine, Nat. Mater. 14, 871 (2015).

${ }^{2}$ S. J. Gong, J. Cai, Q. F. Yao, W. Y. Tong, X. Wan, C. G. Duan, and J. H. Chu, J. Appl. Phys. 119, 125310 (2016).

${ }^{3}$ S. LaShell, B. A. McDougall, and E. Jensen, Phys. Rev. Lett. 77, 3419 (1996).
}

${ }^{4}$ S. J. Gong, C. G. Duan, Y. Zhu, Z. Q. Zhu, and J. H. Chu, Phys. Rev. B 87, 035403 (2013).

${ }^{5}$ Yu. M. Koroteev, G. Bihlmayer, J. E. Gayone, E. V. Chulkov, S. Blügel, P. M. Echenique, and Ph. Hofmann, Phys. Rev. Lett. 93, 046403 (2004).

${ }^{6}$ H. Nakamura, T. Koga, and T. Kimura, Phys. Rev. Lett. 108, 206601 (2012).

${ }^{7}$ J. Nitta, T. Akazaki, H. Takayanagi, and T. Enoki, Phys. Rev. Lett. 78, 1335 (1997).

${ }^{8}$ K. Ishizaka, M. S. Bahramy, H. Murakawa, M. Sakano, T. Shimojima, T. Sonobe, K. Koizumi, S. Shin, H. Miyahara, A. Kimura, K. Miyamoto, T. Okuda, H. Namatame, M. Taniguchi, R. Arita, N. Nagaosa, K. Kobayashi, Y. Murakami, R. Kumai, Y. Kaneko, Y. Onose, and Y. Tokura, Nat. Mater. 10, 521 (2011)

${ }^{9}$ D. Di Sante, P. Barone, R. Bertacco, and S. Picozzi, Adv. Mater. 25, 509 (2013).

${ }^{10}$ A. Stroppa, D. Di Sante, P. Barone, M. Bokdam, G. Kresse, C. Franchini, M. H. Whangbo, and S. Picozzi, Nat. Commun. 5, 5900 (2014).

${ }^{11}$ M. Kim, J. Im, A. J. Freeman, J. Ihm, and H. Jin, Proc. Natl. Acad. Sci. U.S.A. 111, 6900 (2014).

${ }^{12}$ M. Kepenekian, R. Robles, C. Katan, D. Sapori, L. Pedesseau, and J. Even, ACS Nano 9, 11557 (2015).

${ }^{13}$ A. Narayan, Phys. Rev. B 92, 220101(R) (2015).

${ }^{14}$ D. D. Sante, P. Barone, A. Stroppa, K. F. Garrity, D. Vanderbilt, and S. Picozzi, Phys. Rev. Lett. 117, 076401 (2016).

${ }^{15}$ S. Picozzi, Front. Phys. 2, 1 (2014).

${ }^{16}$ S. Datta and B. Das, Appl. Phys. Lett. 56, 665 (1990).

${ }^{17}$ E. Y. Tsymbal and H. Kohlstedt, Science 313, 181 (2006).

${ }^{18}$ V. Garcia and M. Bibes, Nat. Commun. 5, 4289 (2014).

${ }^{19}$ L. L. Tao and J. Wang, Appl. Phys. Lett. 108, 062903 (2016).

${ }^{20}$ M. Tyunina, J. Narkilahti, M. Plekh, R. Oja, R. M. Nieminen, A. Dejneka, and V. Trepakov, Phys. Rev. Lett. 104, 227601 (2010).

${ }^{21}$ H. Nakamura and T. Kimura, Phys. Rev. B 80, 121308(R) (2009).

${ }^{22}$ P. D. C. King, R. H. He, T. Eknapakul, P. Buaphet, S.-K. Mo, Y. Kaneko, S. Harashima, Y. Hikita, M. S. Bahramy, C. Bell, Z. Hussain, Y. Tokura, Z.-X. Shen, H. Y. Hwang, F. Baumberger, and W. Meevasana, Phys. Rev. Lett. 108, 117602 (2012).

${ }^{23}$ K. V. Shanavas and S. Satpathy, Phys. Rev. Lett. 112, 086802 (2014).

${ }^{24}$ D. Vanderbilt, Phys. Rev. B 41, 7892(R) (1990).

${ }^{25}$ P. Giannozzi, S. Baroni, N. Bonini et al., J. Phys.: Condens. Matter 21, 395502 (2009).

${ }^{26}$ J. P. Perdew and A. Zunger, Phys. Rev. B 23, 5048 (1981).

${ }^{27}$ B. Meyer and D. Vanderbilt, Phys. Rev. B 65, 104111 (2002).

${ }^{28}$ L. L. Tao and J. Wang, J. Appl. Phys. 119, 224104 (2016).

${ }^{29}$ M. Gajdoš, K. Hummer, G. Kresse, J. Furthmüller, and F. Bechstedt, Phys. Rev. B 73, 045112 (2006)

${ }^{30}$ Y. Yang, C. S. Lin, J. F. Chen, L. Hu, and W. D. Cheng, J. Appl. Phys. 116, 153709 (2014)

${ }^{31}$ J. H. Haeni, P. Irvin, W. Chang, R. Uecker, P. Reiche, Y. L. Li, S. Choudhury, W. Tian, M. E. Hawley, B. Craigo, A. K. Tagantsev, X. Q. Pan, S. K. Streiffer, L. Q. Chen, S. W. Kirchoefer, J. Levy, and D. G. Schlom, Nature 430, 758 (2004).

${ }^{32}$ R. Wördenweber, E. Hollmann, R. Kutzner, and J. Schubert, J. Appl. Phys. 102, 044119 (2007).

${ }^{33}$ N. A. Pertsev, A. G. Zembilgotov, and A. K. Tagantsev, Phys. Rev. Lett. 80, 1988 (1998).

${ }^{34}$ Y. Zhang, M. Liu, J. Wang, T. Shimada, and T. Kitamura, J. Appl. Phys. 115, 224107 (2014).

${ }^{35}$ Z. Zhong, A. Tóth, and K. Held, Phys. Rev. B 87, 161102(R) (2013).

${ }^{36}$ G. Khalsa, B. Lee, and A. H. MacDonald, Phys. Rev. B 88, 041302(R) (2013).

${ }^{37}$ P. Kim, K. T. Kang, G. Go, and J. H. Han, Phys. Rev. B 90, 205423 (2014).

${ }^{38}$ Z. Zhong, L. Si, Q. Zhang, W.-G. Yin, S. Yunoki, and K. Held, Adv. Mater. Interfaces 2, 1400445 (2015).

${ }^{39}$ P. Löwdin, J. Chem. Phys. 19, 1396 (1951). 